\title{
Commentary on Efficacy of two multimodal treatments on physical strength of occupationally sub grouped male with low back pain
}

\author{
Suraj Kumar* \\ Department of Physiotherapy, Uttar Pradesh University of Medical Sciences, India
}

The author in this study attempted to determine the effect of two treatment protocols that are conventional treatment a combination of two electrotherapy (ultrasound and short wave diathermy) and one exercise therapy (lumbar strengthening exercises) or Dynamic Muscular Stabilization Techniques (DMST) an active approach of stabilizing training on five occupationally sub grouped male LBP (low back pain) patients which made the study interesting as the versatility of the treatments can be seen in different groups. Indeed, the author explores the effects of these treatments on outcome measures pain and physical strength [Back Pressure Changes (BPC) and Abdominal Pressure Changes (APC)].

Occupational are more prone to LBP due to their regular static work postures and workstation design. Compensated LBP is a matter of particular importance, since this is the leading cause of disability for workers under the age of $45 \mathrm{yrs}$ [1]. and the second leading cause of industrial absenteeism [2]. Thus, this study has opened a new dimension in improvement or management of occupational LBP, where work losses, compensation and cost associated with the treatments are very high.

The newness of the study is that the management of sub-acute or chronic nonspecific LBP, through DMST or CONV in seen in heterogeneous occupational population and its long-term effects are recorded which was earlier not done. It should be acknowledged that this study meets the purposes for which it was conducted, as Pain, physical strength (BPC, APC), improved significantly more in patients those receiving DMST as compared to CONV.

The authors believed that the introduction of multimodal treatment program would improve the pain and physical strength of the patient. This was found to be true, as shown by the results in the form of change percentage from pre to post of all the variables.

However, there are few points of discussion that need further investigation. Though the patient showed great improvement but as no follow up was taken in between $0^{\text {th }}$ day and $180^{\text {th }}$ day, it is not possible to determine that the actually improvement is the only effect of exercises.

In this study under conventional treatment ultrasound as well as SWD are used. Both are heating modalities than what was the reason for using two modalities is not clear. As in most of the cases for larger area and for muscular origin pain SWD is preferred most.

The results of this study can be generalized to all occupational LBP patients. However, it is strongly suggested that a similar study should be performed on a larger population as the higher number of subjects either of these subgroups may influence the outcomes of the treatments.

\section{References}

1. Kelsey JL, White AA (1980) Epidemiology and impact of low back pain. Spine (Phila $\mathrm{Pa}$ 1976) 5: 133-142. [Crossref]

2. Kelsey JL, Golden AL (1988) Occupational and workplace factors associated with low back pain. Occup Med 3: 7-16. [Crossref]
Copyright: (C)2018 Kumar S. This is an open-access article distributed under the terms of the Creative Commons Attribution License, which permits unrestricted use, distribution, and reproduction in any medium, provided the original author and source are credited.
${ }^{*}$ Correspondence to: Suraj Kumar, Department of Physiotherapy, Uttar Pradesh University of Medical Sciences, India, E-mail: surajdr2001@yahoo.com

Received: June 08, 2018; Accepted: June 23, 2018; Published: June 27, 2018 